Service social

\title{
Quelques réflexions sur les mauvais traitements et la négligence exercés à l'endroit des personnes âgées
}

\section{Daphné Nahmiash}

Volume 44, numéro 2, 1995

Visages de la violence

URI : https://id.erudit.org/iderudit/706695ar

DOI : https://doi.org/10.7202/706695ar

Aller au sommaire du numéro

Éditeur(s)

École de service social de l'Université Laval

ISSN

1708-1734 (numérique)

Découvrir la revue

Citer cet article

Nahmiash, D. (1995). Quelques réflexions sur les mauvais traitements et la négligence exercés à l'endroit des personnes âgées. Service social, 44(2),

111-128. https://doi.org/10.7202/706695ar
Résumé de l'article

Le présent article passe en revue les écrits décrivant les caractéristiques des aînés, vivant dans la communauté, qui souffrent de mauvais traitements ou de négligence exercés par une personne de confiance. La littérature sur le sujet présente les caractéristiques des aînés qui risquent d'être victimes de mauvais traitements ou de négligence, les caractéristiques des aidants naturels qui sont des agresseurs potentiels et les facteurs sociaux et culturels pouvant mener à de tels abus. L'article suggère que c'est l'interaction entre les traits caractéristiques des aînés, ceux des agresseurs et le contexte d'aide qui contribue à la situation d'abus plutôt que les facteurs individuels. Enfin, l'auteure propose une nouvelle approche pour le travailleur social et des stratégies d'intervention qui redonneraient leur pouvoir aux personnes âgées, leur permettant ainsi de sortir de leur état d'impuissance. 


\section{Quelques réflexions sur les mauvais traitements et la négligence exercés à l'endroit des personnes âgées}

Daphné NAHMIASH

Professeure invitée en travail social, Université McGill Étudiante au doctorat en travail social Université Laval

Durant les années 1960, on s'est intéressé de près au phénomène de la violence à l'égard des enfants, alors que dans les années 1970 c'est plutôt la violence conjugale qui a occupé le devant de la scène. Au cours des décennies 1980 et 1990 les mauvais traitements à l'endroit des personnes âgées ont fait l'objet d'une attention croissante. À la suite de nombreuses études d'abord menées aux États-Unis et en Grande-Bretagne, une prise de conscience du phénomène au Canada a entraîné en 1989 la réalisation d'une première étude nationale par Podnieks et al. La même année, au Québec, le Comité sur les abus exercés à l'endroit des personnes âgées publiait son premier rapport détaillé, Vieillir... en toute liberté (Gouvernement du Québec, 1989). 
Les aînés peuvent être victimes de mauvais traitements et de négligence chez eux, en milieu d'hébergement ou encore dans la rue, par le fait d'une personne inconnue. Nous nous intéresserons ici particulièrement aux mauvais traitements et à la négligence que font subir aux aînés vivant dans leur milieu naturel des aidants non rémunérés (surtout des membres de la famille), qui occupent une position de confiance. Nous ferons une revue de la documentation sur le sujet, afin de déterminer l'étendue du problème, les caractéristiques des victimes, celles des agresseurs et les explications les plus répandues sur leur comportement. En conclusion, nous suggérerons aux travailleurs sociaux des façons d'utiliser ces connaissances dans leur pratique pour reconnaître les personnes âgées victimes de violence, pour empêcher de telles situations d'abus de pouvoir de se produire ou de se poursuivre et pour aider les victimes à sortir de cette situation.

\section{UNE DÉFINITION DES MAUVAIS TRAITEMENTS}

Les auteurs du rapport du gouvernement du Québec ont adopté la définition de violence exercée à l'endroit d'une personne âgée qu'a donnée Michot (1973): une action directe ou indirecte destinée à porter atteinte à une personne ou à la détruire, soit dans son intégrité physique ou psychique, soit dans ses possessions, soit dans ses participations symboliques (Gouvernement du Québec, 1989).

Les différentes formes de mauvais traitements ont été définies dans le rapport comme étant la violence physique, qui inclut la violence sexuelle et le recours à la douleur physique, aux brûlures, à la rudesse; la violence psychologique ou émotive, qui comprend la violence verbale, les menaces, I'infantilisation, I'humiliation et l'isolation ou la privation de chaleur humaine; I'exploitation financière, dont les principaux éléments sont I'utilisation inadéquate de l'argent ou des biens d'une personne et la fraude. La négligence comporte une forme active et une forme passive, selon que l'acte est intentionnel ou non, et implique l'omission de fournir des soins essentiels. L'auto-négligence est aussi rapportée comme une forme de violence, mais qui diffère des autres en ce qu'elle ne concerne que soi. Nous n'en parlerons donc pas davantage ici. Cependant, il peut arriver que des membres de la famille ou des amis constatent ce type de violence et $n^{\prime} y$ réagissent pas. La violation des droits, qui a également été définie comme de la violence, se présente comme le fait d'empêcher une personne âgée de prendre ses propres décisions, 
en la forçant par exemple à aller demeurer dans une résidence pour aînés. Enfin, la violence sociale ou collective a été définie comme une forme de violence sociétale fondée sur l'âgisme et d'autres façons de traiter les personnes âgées qui affectent leur dignité et leur identité propre.

Bien que la majorité des études sur les mauvais traitements à l'endroit des personnes âgées utilisent les catégories de violence physique, psychologique et financière et de négligence, la plupart des études sur le sujet constatent la difficulté des chercheurs à trouver une définition commune des mauvais traitements. Ainsi, Stones (1991) estime que s'il est tellement difficile de définir les mauvais traitements auxquels on soumet des personnes âgées, $c^{\prime}$ est que le sens varie selon la personne qui définit et la raison pour laquelle elle le fait. Les différents sens dépendent de la source de la définition et du contexte dans lequel on I'utilise. Stones mentionne également que les formes de mauvais traitements peuvent être classées en fonction de standards législatifs, organisationnels ou normatifs. Les standards normatifs de la communauté quant aux mauvais traitements sont les plus difficiles à définir; ils diffèrent selon le bagage culturel de la personne et constituent un indicateur des attitudes à l'égard de la violence en général. Stones a relevé un large consensus parmi les aînés et les intervenants autour d'éléments qui indiquent une violence plus ou moins grande, et les éléments classés comme les plus violents étaient surtout des manifestations de violence physique.

Très peu de publications se sont intéressées jusqu'à maintenant à la façon dont les personnes âgées chez les autochtones et les minorités ethniques définissent la violence et la négligence (Dow Pittaway et al., 1995). La plupart des recherches sur la violence en ignorent les aspects culturels et semblent supposer que toutes les personnes âgées sont semblables dans leurs attitudes et leurs perceptions. II va de soi qu'une recherche plus poussée où l'accent porterait sur la diversité ethnique et régionale est encore à faire.

Thériault (1994) s'est intéressé récemment à la manière dont un groupe de personnes du troisième âge dans un centre de jour (qui n'étaient pas victimes de violence) définissaient le mauvais traitement. Elles ont confirmé que:

les abus et la violence sont inscrits dans nos rapports, dans nos échanges interpersonnels et dans nos organisations. Le problème de la violence dans nos rapports interpersonnels est tellement tabou que nous devons apprendre à décoder le langage, nommer l'innommable et avant toute chose offrir des lieux et des moments pour échanger sur de telles situations dans un langage clair et transparent pour [elles] (p. 96). 


\section{L'AMPLEUR DU PROBLÈME}

On a souvent fait état de l'ampleur du phénomène des mauvais traitements. Une certaine confusion persiste cependant dans les écrits sur le sujet en ce qui a trait à la définition de la prévalence et de I'incidence de l'abus de pouvoir (Kozack, Elmslie et Verdon, 1995). L'incidence résulte de la fréquence des nouveaux cas durant une période donnée au sein d'un groupe à risque dont les membres n'avaient pas été victimes de violence ou de négligence jusque-là. Quant à la prévalence, elle fournit une indication de l'ampleur du problème actuel et ne peut être utilisée pour prévoir le nombre de cas qui surviendront dans le futur (Kozack et al., 1995). Kozack et al. rapportent les données qui suivent sur la prévalence des mauvais traitements au Canada.

La première étude recensée au Canada est celle menée au Québec par Bélanger et al. (1981). Cette recherche, qui s'appuie sur un faible taux de réponse de $32 \%$, rapportait la présence de violence physique dans une proportion de $21 \%$ de la clientèle âgée des travailleurs sociaux, celle de violence psychologique dans $55 \%$ des cas, d'exploitation financière chez $24 \%$ des personnes âgées et de violation des droits chez $25 \%$. Des résultats similaires ont été obtenus par Shell (1982), qui a interrogé 105 professionnels d'organismes de services de santé au Manitoba. Ces études ont été suivies de rapports sur des visites à un hôpital de Winnipeg par King en 1984 et d'une étude de cas par des intervenants (Lamont, 1985); d'une étude menée en Alberta par Stevenson (1985), d'une étude du ministère des Services sociaux et communautaires de I'Ontario (1986) et d'études de données par Grandmaison (1988).

Seule l'étude nationale de Podnieks a été suffisamment rigoureuse dans son expérimentation pour évaluer la prévalence réelle au Canada de la violence et de la négligence parmi les personnes âgées vivant dans la communauté. Cette étude rapporte que le taux de prévalence de la violence et de la négligence chez les aînés est de $4 \%$ et qu'il varie légèrement selon les régions du pays (4\% au Québec). L'exploitation financière $(2,5 \%)$, qui porte sur l'argent ou les biens, et l'agression verbale constante $(1,4 \%)$ sont les cas les plus fréquemment rapportés par les victimes elles-mêmes. Une étude récente menée au niveau national par Dow Pittaway et al. (1995) a fait ressortir, à partir d'un échantillon de 489 dossiers de victimes de violence connues, que la violence psychologique correspondait à 40,7\% des mauvais traitements subis par les personnes âgées, suivie de la violence physique $(28,6 \%)$, de l'exploitation financière $(26,6 \%)$ et de la négligence $(12,1 \%)$. 
Enfin, une étude québécoise menée localement sur le territoire du CLSC Notre-Dame-de-Grâce - Montréal-Ouest, surtout parmi les usagers du programme de soutien à domicile du CLSC, a relevé chez 421 personnes âgées une incidence de mauvais traitements se situant entre $7,1 \%$ et $10,4 \%$, ainsi qu'il a été noté par les intervenants au sein de la clientèle d'aidants naturels et de bénéficiaires du CLSC (Reis, Nahmiash et Shrier, octobre 1993). Les formes de violence exercée par les aidants naturels semblaient être de nature psychosociale ou financière, tandis que la violence exercée par des bénéficiaires, qui constituait seulement $3 \%$ des cas de mauvais traitements, paraissait surtout de nature psychosociale.

\section{LES PROFILS DES VICTIMES ET DE LEURS AGRESSEURS}

Nous avons recensé la documentation sur le sujet, afin de rapporter la façon dont les aînés victimes de violence ainsi que leurs agresseurs y sont décrits, et nous avons mis en évidence les enjeux principaux. Bien que nous ne puissions malheureusement pas exposer en détail dans cet article les résultats de nos recherches, nous présentons une liste de caractéristiques des personnes âgées victimes de mauvais traitements relevées au cours de notre dernière revue des écrits (Kosberg et Nahmiash, 1996). Ce sont surtout des femmes, en majorité des veuves, qui ont en général une mauvaise santé, sont d'un âge avancé, qui éprouvent des problèmes de toxicomanie, ont des conditions de logement précaires (ex. : manque d'intimité). On note également des facteurs psychologiques ou personnels, comme la dépression, la présence de comportements perturbateurs causés par la démence, la dépendance, I'isolement et le manque de soutien social, de même que des attentes irréalistes à l'égard du soignant naturel.

En ce qui a trait aux auteurs de mauvais traitements, nous avons relevé chez eux ces traits caractéristiques au cours de la même recension : problèmes de toxicomanie; troubles mentaux ou émotifs; manque d'expérience en matière de soins à donner; répugnance à donner des soins; passé de violence; dépendance à l'égard de la personne qui reçoit les soins; confusion, démence, stress et épuisement occasionnés par les soins à fournir; traits de la personnalité tels que contrôle, blâme, critique excessive et absence de compassion; manque de soutien social.

De plus, certains aspects du contexte social des soins sont également caractéristiques des situations de mauvais traitements. II s'agit des difficultés financières de la famille, de la pauvreté des relations sociales et des pressions ressenties quant à la nécessité 
d'être productif, de la violence familiale, d'un soutien social déficient, de la discorde dans la famille, des conditions de logement précaires (ex.: promiscuité), de la transmission de la violence d'une génération à l'autre, de même que du fardeau que constituent les soins pour les membres de la famille et leur désir d'hébergement en milieu institutionnel.

Enfin, certaines normes, attitudes et valeurs culturelles semblent avoir une influence sur les mauvais traitements envers les personnes âgées. Il en est ainsi de l'âgisme, du sexisme, de certaines attitudes culturelles à l'égard de la violence, des réactions à un comportement abusif, des attitudes envers les personnes handicapées et des obligations relatives aux soins à donner aux membres de la famille. Les différentes études sur le sujet révèlent qu'il existe un débat à savoir si la violence est causée par la dépendance de la victime (Quinn et Tomita, 1986) ou par la dépendance de l'agresseur (Pillemer, 1986). Pillemer en vient à la conclusion qu'il est bien possible que ce soit une interaction de facteurs entre la victime âgée et l'aidant naturel agresseur, aussi bien que le contexte social et culturel, qui contribue à la violence, plutôt que des facteurs liés uniquement à la victime ou à I'agresseur. Les travailleurs sociaux doivent donc être en mesure de reconnaître les traits caractéristiques des victimes et des agresseurs ou agresseurs potentiels; ils doivent aussi pouvoir évaluer le contexte social, familial et culturel qui influe sur le comportement familial. Tous ces éléments sont illustrés dans la figure de la page suivante.

Étant donné que la plupart des actes de violence et de négligence sont commis au domicile de la personne âgée par des aidants naturels (Douglass, 1983), et bien que la plupart des aidants naturels ne soient pas des agresseurs, il est important que les travailleurs sociaux non seulement trouvent des façons de parler de ce problème délicat dans des termes qui conviennent aux personnes âgées et aux aidants naturels, mais aussi qu'ils comprennent le contexte entourant la prestations des soins.

\section{LES AIDANTS NATURELS ET LE CONTEXTE D'AIDE}

De nombreuses études ont décrit les personnes qui prodiguent des soins aux personnes âgées. Les aidants naturels sont définis comme des personnes qui soignent une personne âgée vulnérable, habituellement un membre de la famille, un ami, ou un voisin, qui a perdu une certaine partie de son autonomie à la suite d'une dégradation physique ou mentale (Conseil consultatif national sur le troisième âge, 1991). 


\section{Interrelation des traits caractéristiques des victimes et des agresseurs ainsi que du contexte d'aide}

\section{Contexte social}

Difficultés financières, violence familiale, absence de soutien social, mésententes familiales, conditions de logement, transmission de la violence d'une génération à l'autre
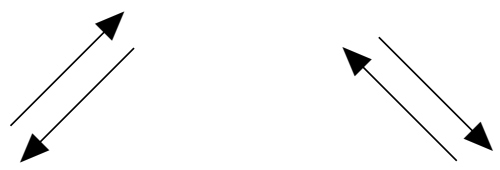

\section{Victime}

Sexe (femmes)

État civil (veuves)

Santé (mauvaise)

Âge avancé

Toxicomanie

Conditions de logement

Facteurs psychologiques

(dépression)

Comportements perturbateurs

Dépendance, isolement

Manque de soutien social

Attentes irréalistes

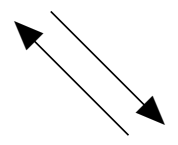

\section{Agresseur}

Toxicomanie

Troubles mentaux ou émotifs

Manque d'expérience

dans les soins

Répugnance à donner les soins

Passé de violence

Dépendance à l'égard

de la personne âgée

Confusion, démence

Stress et épuisement

Traits personnels

Manque de soutien social

\section{Normes culturelles}

Âgisme, sexisme, attitudes culturelles à l'égard de la violence, réactions à un comportement abusif, attitudes à l'égard des personnes invalides, contexte familial des soins à donner 
Il y a trois fois plus de femmes que d'hommes parmi les aidants naturels. La plupart sont les épouses ou les femmes des personnes qui reçoivent les soins. Toutefois, des données américaines révèlent qu'environ $30 \%$ des aidants naturels sont des hommes (Stone et al., 1987). On peut en déduire que le tableau des soins pourrait changer chez nous aussi dans un avenir plus ou moins rapproché.

Selon Rosenthal (1987), le mythe selon lequel les familles abandonnent les aînés, mythe que les médias contribuent à répandre, doit être combattu avec vigueur par les parents âgés et leurs enfants qui comptent encore les uns sur les autres, se fréquentant et s'entraidant, particulièrement dans les périodes de crise.

Étant donné que la taille des familles diminue au Canada, Baker (1987) insiste sur le fait que les enfants sont moins nombreux à pouvoir s'occuper de leurs parents qu'ils l'étaient il y a quelques générations, même si une maisonnée comptant trois générations a toujours été exceptionnelle en Amérique du Nord (Laslett, 1983; Synge, 1980). La situation est très différente au Japon où près des deux tiers des personnes âgées vivent encore dans une maison où logent trois générations et où seulement $1,6 \%$ des adultes vivent dans un établissement comparativement à $7 \%$ au Québec (Rochon, 1994).

Au Québec, une importante étude par Garant et Bolduc (1990) a mené aux conclusions suivantes sur les soins à la famille:

- Aujourd'hui plus que jamais, la famille constitue la plus importante source d'aide (p. 11).

- Aussi longtemps que l'état de santé de la personne n'est pas trop détérioré, le réseau familial fonctionne comme un modèle de réciprocité: on donne d'un côté comme de l'autre. C'est la famille plutôt que le système de santé et de services sociaux qui agit comme principale source d'aide lorsque l'état de santé d'un de ses membres se détériore gravement (p.12). Considérant les nouvelles pressions démographiques, le rôle changeant des femmes et les conditions de logement, nous ne pouvons être certains que les familles pourront continuer d'assumer leur rôle actuel de fournisseur de soins.

- Les aidants naturels sont surtout des femmes qui, pour la plupart, assument leurs responsabilités seules, sans I'aide du système public (p. 38). Le soutien familial se caractérise par sa diversité : il consiste en un soutien émotif, une aide financière, un rôle d'intermédiaire entre le système de soins institutionnel et la personne âgée, de nombreux services d'ordre pratique et I'assurance d'une présence continue. La tâche est très lourde et la durée de la situation d'aide très longue, ce qui constitue une 
source constante de stress et de tension émotive. La situation impose aussi des contraintes financières. Le soutien aux personnes souffrant de démence est particulièrement difficile, et c'est de loin la tâche qui occasionne le plus de stress.

Lesemann et Chaume (1989) ont trouvé qu'une relation basée sur les soins apparaît comme un phénomène relativement typique, qui comporte des dimensions émotives et morales issues d'une histoire complexe de relations familiales; les familles caractérisées par la spécificité de leurs rapports et de leurs pratiques comptent sur I'État pour jouer un rôle essentiel sur lequel elles exerceraient un contrôle. De même, Mace et Rabins (1981) décrivent une situation de soins auprès d'une personne atteinte de démence comme une journée de 36 heures et de nombreuses études par Maheu et Guberman (1992) concluent que la prestation de soins à un parent dépendant est un travail qui requiert des conditions particulières et une organisation complexe de ressources et d'autres formes de soutien. De plus, les aidants naturels ne sont pas nécessairement outillés sur le plan de la formation, de la supervision ou des conditions nécessaires pour acquérir les compétences voulues. Le secteur public ne répond toutefois pas forcément au besoin de ressources et de soutien supplémentaires des aidants naturels: pensons aux compressions budgétaires, au mouvement vers la privatisation et à une tendance croissante à compter sur la famille pour les soins à donner (Stryckman et Nahmiash, 1994).

L'étude menée par Chappell (1989) au Canada ajoute que même lorsque les ressources existent vraiment, les aidants naturels ne recourent aux services formels (maison de soins) qu'en dernier ressort, une fois toutes leurs propres ressources épuisées. L'étude signale également que lorsque les personnes soignantes demandent de l'aide, c'est qu'elles sont rendues au bout du rouleau, que l'état de santé de la personne qu'elles soignent s'est gravement détérioré, que celle-ci est peut-être même parvenue au stade terminal de sa maladie.

Nous pouvons conclure de ce qui précède que les soins informels donnés aux aînés vulnérables par des membres de la famille occupent une grande place, qu'ils sont épuisants et que les relations et les pratiques liées à l'aide sont propres à chacun. Cependant, le contexte social de l'aide, associé à d'autres facteurs qui caractérisent la victime et l'auteur de mauvais traitements, peut favoriser dans certains cas un comportement abusif. En fait, selon Kosberg (1983), ceux qui font les placements se tournent peut-être trop rapidement, dans leur désir de trouver une solution facile et peu coûteuse pour les soins à donner à une personne âgée, vers les membres de la famille sans évaluer les compétences de la famille ni la pression que celle-ci 
subira. On peut également noter le peu de soutien fourni par l'État aux aidants naturels qui, tout bien considéré, apportent une contribution majeure à l'aide familiale.

C'est dans ce contexte précaire de personnes âgées devenues vulnérables et qui dépendent pour leurs soins de membres de leur famille ou d'autres personnes de leur réseau informel qu'on relève souvent des cas de mauvais traitements et de négligence de personnes âgées (Douglass, 1983).

\section{CAUSES DES MAUVAIS TRAITEMENTS ET DE LA NÉGLIGENCE À L'ENDROIT DES PERSONNES ÂGÉES}

Les causes des mauvais traitements exercés par des aidants naturels semblent complexes et multiples. On peut regrouper les principales explications relevées dans les écrits à l'intérieur des catégories suivantes: le modèle situationnel, la théorie de l'échange social et I'interaction symbolique. McDonald et al. (1991) décrivent ainsi ces approches:

Le modèle situationnel suggère que le mauvais traitement est une réponse irrationnelle aux conditions environnementales et à une crise situationnelle. Les variables situationnelles comprennent: 1. les facteurs associés à la personne soignante; 2 . les facteurs associés à la personne âgée; 3 . les facteurs sociostructurels. Ces trois groupes de facteurs ne s'excluent pas nécessairement les uns les autres. Les facteurs liés à la personne soignante peuvent venir d'une violence transmise d'une génération à l'autre ou apprise, de traits de la personnalité, du tissu des dépendances, $d^{\prime}$ une crise familiale et d'un stress personnel. Les facteurs liés aux personnes âgées semblent provenir surtout des dépendances, bien qu'ils puissent aussi résulter d'une violence transmise d'une génération à l'autre ou d'un stress personnel. Les facteurs sociostructurels peuvent comprendre des stresseurs extérieurs et des attitudes sociétales à l'égard des personnes âgées.

La théorie de l'échange social, selon les mêmes auteurs, pose que les gens âgés, à mesure qu'ils vieillissent, ont moins accès au pouvoir, qu'ils disposent de moins de ressources et sont progressivement moins capables d'exécuter des tâches instrumentales. Plus ils avancent en âge et plus le déséquilibre s'accroît; les personnes âgées deviennent généralement plus impuissantes, plus dépendantes et plus vulnérables que leurs aidants naturels. 
L'interaction symbolique est un processus qui implique au moins deux personnes. Elle se produit dans le temps, comporte des phases identifiables qui se répètent et sont interreliées et commande des négociations et des renégociations constantes. Dans cette approche, liée à l'apprentissage social, les mauvais traitements sont vus comme un phénomène récurrent dans la famille, un phénomène cyclique associé à un passé de relations violentes dans la famille (Shell, 1982).

Aucun modèle théorique ne semble fournir une explication d'ensemble complète sur le phénomène des mauvais traitements envers les personnes âgées (Phillips, 1986). Il existe par ailleurs une approche théorique et pratique moins connue et moins utilisée, basée sur la théorie de l'empowerment. L'empowerment a été défini (Santé Canada, 1993) comme un processus par lequel on aide les individus à accroître au maximum leur confiance en eux, leurs compétences et leurs habiletés, afin qu'ils puissent exercer un contrôle sur leur vie et prendre des décisions qui sont dans leur meilleur intérêt. L'empowerment comporte aussi un élément de choix, avec des options disponibles, accessibles. Une analyse récente révèle que de nombreuses études s'appuyant sur la théorie de l'empowerment ont relevé I'impuissance comme trait caractéristique des personnes (Lord et McKillop, 1990). On a identifié par ailleurs la rupture sociale comme cause inhérente de l'impuissance (Myers, 1993). Myers, dans sa recherche auprès de personnes âgées, résume ce processus en quatre stades résultant en des personnes qui ne croient pas avoir une chance de remporter une victoire ou de résoudre un problème. On a associé cet état d'impuissance avec les personnes âgées victimes de mauvais traitements (Blunt, 1993; Myers, 1993), et l'empowerment a été proposé comme stratégie d'intervention pour aider les victimes à se libérer de leur état d'impuissance.

À la lumière de ces modèles théoriques et par suite de I'application de certains de leurs éléments dans le projet CARE, une étude de trois ans menée par le CLSC Notre-Dame-de-Grâce - MontréalOuest (Reis et Nahmiash, 1995), il semble que les mauvais traitements ne soient pas attribuables surtout à la vulnérabilité ou à la dépendance des personnes âgées, comme le supposent certaines études (Kosberg, 1986 ; Quinn et Tomita, 1986), mais à certains facteurs interreliés qui caractérisent I'aidant naturel (Pillemer, 1986; Thériault, 1994; Reis et al., novembre 1993) aussi bien que le contexte social. Les théories qui mettent l'accent sur la vulnérabilité et la dépendance ou encore sur le comportement provocateur des personnes âgées pourraient aussi mettre en avant la notion qui consiste à blâmer la victime. 
Dans la recherche du projet CARE (Reis et al., novembre 1993), on a relevé 22 indicateurs de risque parmi un nombre plus grand d'indicateurs associés aux personnes soignantes et aux personnes soignées, afin de distinguer les aidants naturels violents de ceux qui ne l'étaient pas. On a alors trouvé que les différences les plus marquées se situaient entre les facteurs caractérisant les aidants et ceux caractérisant les personnes soignées.

Les traits caractéristiques de l'aidant qui étaient le plus typiques des mauvais traitements étaient liés aux comportements inappropriés (par exemple, entrer de force une cuillère dans la bouche d'une personne atteinte de démence qui refuse de manger), au manque d'expérience et d'empressement de l'aidant naturel, aux troubles mentaux et affectifs, à l'abus de drogues ou d'alcool, de même qu'aux facteurs associés au contexte social de l'aide.

La validation ultérieure d'un outil de dépistage (CASE) utilisé dans le projet CARE (Reis et Nahmiash, 1995), administré à des aidants naturels à qui on a posé huit questions autour du comportement abusif, a suggéré que les aidants qui éprouvaient plus de difficulté à percevoir leurs problèmes de contrôle de soi, qui étaient malheureux et dépressifs et dont la santé mentale et physique n'était pas bonne montraient une plus grande tendance à maltraiter leurs parents dépendants que les autres personnes aidantes vivant une situation similaire.

Pour conclure cette revue des écrits, disons qu'une recherche plus approfondie s'impose afin de trouver des explications aux situations de mauvais traitements. De plus, les travailleurs sociaux doivent remettre constamment en question leurs propres façons de voir et lire la documentation sur le sujet afin de se garder au fait des nouvelles informations susceptibles de guider leur action. Les chercheurs aussi bien que les intervenants devraient s'intéresser aux interactions entre les caractéristiques de la personne soignante et de la personne soignée comme étant des éléments contributifs aux situations de violence. Cependant, le contexte d'aide, le rôle de l'État dans le soutien aux aidants naturels et les relations entre les personnes qui maltraitent et leurs victimes peuvent aussi être des facteurs contributifs.

\section{STRATÉGIES D'INTERVENTION}

À la lumière de l'information rapportée plus haut, que peut faire un travailleur social pour prévenir les mauvais traitements ou pour aider les victimes à résoudre leurs problèmes ? On a pu classer les programmes de services au Canada en trois catégories: programmes de protection 
destinés aux adultes, programmes contre la violence familiale et programmes de défense des droits (McDonald et al., 1991). Les stratégies d'intervention, selon Dow Pittaway et al. (1995), portent sur les services suivants :

- Éducation (du public, des professionnels, des adultes à risque, intergénérationnelle).

- Promotion de la santé (groupes d'entraide).

- Prise en charge (évaluation, aiguillage vers les spécialistes appropriés, counseling).

- Thérapie familiale.

- Services juridiques / défense des droits / information.

- Services médicaux.

- Stratégies adaptées aux communautés culturelles.

- Soutien aux aidants naturels (ex.: centre de jour pour adultes, services de soutien à domicile, aide à court terme, programmes de répit).

- Gestion de crise (ex. : refuge d'urgence).

- Coordination des services.

On a relevé de nombreux modèles d'intervention au Québec, par exemple le modèle utilisé dans le projet CARE au CLSC NotreDame-de-Grâce - Montréal-Ouest (Reis et Nahmiash, 1995), qui s'appuie sur des équipes multidisciplinaires et sur une équipe interdisciplinaire de spécialistes (avocats, policiers, spécialistes en santé mentale, experts financiers, représentants de mouvements de défense des droits de la personne et du curateur public). Ce modèle s'appuie également sur une équipe de bénévoles, amis et intervenants, sur un groupe de prise en charge par les victimes, sur des groupes de soutien aux familles des victimes et des agresseurs, de même que sur un comité communautaire dont le mandat est de sensibiliser la population au phénomène des mauvais traitements et de la négligence à l'endroit des personnes âgées.

Une évaluation des interventions de ce programme révèle que ce sont les stratégies dirigées vers la personne et celles basées sur la formation et le counseling des aidants qui ont été parmi les mieux reçues et qui ont le mieux réussi (Nahmiash et Reis, non publié). Cela indique bien que les travailleurs sociaux doivent s'intéresser aux personnes violentes aussi bien qu'à leurs victimes. Les stratégies de prise en charge (empowerment) comme la formation d'un groupe de victimes, qui aident la victime à s'affirmer en développant son estime de soi et en refusant par le fait même le contrôle de l'agresseur, sont 
également apparues très efficaces. Voici quelques exemples de tâches à remplir, relevés par les travailleurs sociaux du CLSC dans les groupes cibles du projet CARE (Reis et Nahmiash, 1995):

- Établir et maintenir le contact.

- Construire une relation de confiance.

- Faire une évaluation de la personne âgée et du soignant (au moyen d'outils et de mesures appropriés).

- Affecter un second intervenant si un conflit d'intérêts éclate dans le travail avec un couple personne soignée - personne soignante.

- Définir et mettre en œuvre des stratégies de prise en charge pour mettre un terme aux mauvais traitements.

- Maintenir des liens et la communication avec les autres membres de l'équipe et les experts.

- Préciser la nature des mauvais traitements et les conséquences du comportement abusif.

- Demeurer à l'affût d'une violence possible ou d'une reprise des mauvais traitements.

- Confronter la personne violente au moment opportun.

- Soutenir la victime dans les procédures judiciaires au besoin.

- Faciliter la prise en charge (empowermentl) de la victime soit en la dirigeant vers un groupe de responsabilisation, soit en recourant à d'autres moyens.

- Fournir directement de l'aide, de l'information et une éducation appropriées à la victime et à l'aidant naturel.

- Défendre la victime et l'informer de ses droits.

- Mettre sur pied les services nécessaires, comme des services de répit ou de soins à domicile.

- Orienter vers des programmes de traitements spéciaux, par exemple des programmes liés à la toxicomanie ou à la santé mentale.

- Mettre la personne en rapport avec un compagnon volontaire.

On a par ailleurs identifié des barrières à la prestation de services dans les écrits sur le sujet. Ainsi, selon Wolf et Pillemer (1994), les intervenants dans les agences peuvent éprouver des difficultés en raison de la fragmentation des services, de la résistance des victimes à accepter de recevoir des services et du manque de personnel formé à cet effet. Les mêmes auteurs ont cependant relevé trois facteurs de réussite dans une recherche fondée sur quatre modèles: se trouver 
dans un grand espace urbain, disposer d'un financement suffisant et bénéficier d'une coopération entre agences. D'autres critères de réussite pour les projets résidaient dans leur coût relativement bas, leur flexibilité, I'information et l'assistance techniques disponibles pour la mise en œuvre de programmes. Toutes les évaluations de programmes recensées dans les écrits semblaient privilégier une approche fondée sur une équipe multidisciplinaire dont les membres peuvent se pencher sur les nombreux et complexes problèmes d'ordre éthique et culturel associés aux situations de mauvais traitements.

Dow et Pillemer (1995) rapportent pour leur part que les victimes se heurtent à des obstacles lorsqu'il s'agit de chercher de I'aide: un réseau de soutien restreint, la peur de perdre leur dignité, le manque d'accessibilité des services, l'absence de moyens de transport, la crainte de la non-confidentialité et la peur des représailles.

\section{CONCLUSION}

Nous pouvons conclure de cette revue globale de la littérature sur le sujet que les mauvais traitements et la négligence constituent un problème sérieux pour les personnes âgées vivant dans la communauté. Ces cas devraient être dépistés par les travailleurs sociaux dans leur pratique grâce à une bonne connaissance des écrits et à I'utilisation des outils et des indicateurs appropriés. Les stratégies visant la prévention des mauvais traitements et de la négligence doivent s'intéresser aux agresseurs et agresseurs potentiels aussi bien qu'à leurs victimes réelles ou potentielles. La politique sociale doit aussi porter sur le contexte d'aide qui peut contribuer à la situation de mauvais traitements et de négligence en ne fournissant pas suffisamment de services, de ressources et de soutien aux personnes qui donnent et qui reçoivent les soins.

\section{Références bibliographiques}

BAKER, M. (1987). Aging in Canadian Society: A Survey. Toronto : McGrawHill Ryerson.

BÉlANGer, L., T. D'ARChe, H. de RAVINEL et P. GRENIER (1981). L'envers du crime. Étude criminologique. Centre international de criminologie comparée. Montréal : Université de Montréal, Les Cahiers de recherche criminologique. 
BLUNT, A.P. (1993). "Financial exploitation of the incapacitated: Investigation and remedies ", Journal of Elder Abuse \& Neglect, vol. 5, n $1: 19-32$.

BRECKMAN, R.S. et R.D. AdELMAN (1988). Strategies for Helping Victims of Elder Mistreatment. Beverley Hills: Sage Publications Inc.

CHAPPELL, N. (1989). Formal programs for informal caregivers of the elderly in supporting elder care. Winnipeg: University of Manitoba.

CONSEIL CONSULTATIF NATIONAL SUR LE TROISIÈME ÂGE (1991). The NACA position on informal caregiving. Ottawa: Approvisionnements et Services Canada.

DOUGLASS, R.L. (1983). "Domestic neglect and abuse of the elderly. Implications for research and service », Journal of Applied Family and Child Studies, $\mathrm{n}^{\circ} 32$ : 395-402.

Dow Pittanay, E., E. Gallagher, M. Stones, D. Nahmiash, J. KosberG, L. STRAIN et J. BOND (1995). Report on services for abused older Canadians. British Columbia: Center on Aging, University of Victoria.

GARANT, L. et M. BOLDUC (1990). L'aide par les proches: Mythes et réalités. Québec: Ministère de la Santé et des Services sociaux.

GOUVERNEMENT DE L'ONTARIO (1986). A report on elder abuse. Toronto: Ontario Advisory Council on Senior Citizens.

GOUVERNEMENT DU QUÉBEC (1989). Vieillir... en toute liberté. Rapport du Comité sur les abus exercés à l'endroit des personnes âgées. Québec : Ministère de la Santé et des Services sociaux.

GOUVERNEMENT DU QUÉBEC (1991). "Vers un nouvel équilibre des âges », Rapport du groupe d'experts sur les personnes aînées.

Grandmaison, A. (1988, octobre). Protection des personnes âgées: Étude exploratoire de la violence à l'égard de la clientèle des personnes âgées du CSSMM. Centre des services sociaux du Montréal métropolitain, Direction des services professionnels.

KING, N.R. (1984). "Exploitation and abuse of older family members: An overview of the problem », dans Joseph J. Costa (dir.), Abuse of the elderly: A guide to resources and services. Lexington, Mass.: Lexington Books, Health.

KOSBERG, J.I. (1983). "The special vulnerability of elderly parents», dans J.I. Kosberg (dir.), Abuse and maltreatment of the elderly: Causes and interventions. Boston: John Wright - PSG.

KOSBERG, J.I. (1986). "Understanding elder abuse: An overview for primary care physicians», dans R.J. Ham (dir.), Geriatric Medicine Annual, p. 114-127. New York: Medical Economic Books.

KOSBERG, J.I. et D. NAHMIASH (à paraître en 1996). "Characteristics of victims and perpetrators and milieus of abuse and neglect», dans L.A. Baumhover et S.C. Beal (dir.), Assessing Elder Abuse in Health Care Settings. Baltimore, MD : Health Professions Press.

KOZACK, J., T. ELMSLIE et J. VERDON (1995). "Perspectives épidémiologiques des mauvais traitements et de la négligence à l'endroit des personnes âgées: revue des textes de recherche nationale et internationale », dans M. MacLean (dir.), Mauvais traitements auprès des personnes âgées : stratégies de changement, p. 174-190. Montréal: Éd. Saint-Martin. 
LAMONT, C. (1985). "La violence à domicile faite aux femmes âgées.» Travail présenté pour le cours EAN6670: Condition féminine et éducation continue. Université de Montréal.

LASLetT, P. (1983). The World We Have Lost., 3e éd. New York: Scribner.

Lesemann, F. et C. Chaume (1989). Familles-Providence: la part de l'État. Montréal : Éd. Saint-Martin.

LORD, J. et MCKILLOP FARLOW (1990, automne). "Une étude sur I'habilitation: répercussions sur la promotion de la santé », Promotion de la santé, p. 2-8.

MACE, N.L. et P.V. RABINS (1981). The 36-hour day. Baltimore: The John Hopkins University Press.

MCDOnAlD, P.L., J.P. HORNiCK, G.B. RobertSOn et J.E. WALLACE (1991). Elder Abuse \& Neglect in Canada. Toronto: Butterworths Canada Ltd.

MAHEU, P. et N. GUBERMAN (1992). "Familles, personnes adultes dépendantes et aide naturelle entre le mythe et la réalité ", La Revue internationale d'action communautaire, 28/68: 40-51.

MICHOT, Y.A. (1973). La violence. Paris: PUF.

MYERS, J.E. (1993). "Personal empowerment», Aging International, p. 3-8.

NAHMIASH, D. (1994, novembre). «A Comparison of Social Welfare Services in the Community in Japan with Quebec », Report to the Heiwa Nakajima Foundation, Tokyo.

NAHMIASH, D. et M. ReIS (non publié). Most successful intervention strategies for abused seniors. Montréal : Projet CARE.

NunNALlY, J.C. Jr. (1959). Test and Measurements. New York: McGraw-Hill Book Company.

PHILLIPS, L.R. (1986). "Theoretical explanations of elder abuse: Competing hypotheses and unresolved issues », dans K.A. Pillemer et R.S. Wolf (dir.), Elder Abuse: Conflict in the Family, p. 197-217. Dover, Mass.: Auburn House Publishing Co.

PILLEMER, K.A. (1986). "Risk factors in elder abuse: Results from a casecontrol study », dans K.A. Pillemer et R.S. Wolf (dir.), Elder Abuse: Conflict in the Family. Dover, Mass. : Auburn House Publishing Co., p. 239-263.

PILlEMER, K.A. et J. SUITOR (1992). «Violence and violent feelings: What causes them among family caregivers?», Journal of Gerontology, vol. 47, $\mathrm{n}^{\circ} 4$ : S165-S172.

Podnieks, E., K.A. Pillemer, J. NiChOlson, J. Shillington et A. Frizzell (1989). A national survey on abuse of the elderly in Canada: Preliminary findings. Toronto: Office of Research and Innovation, Ryerson Polytechnical Institute.

QuINN, M.J. et S.K. TOMITA (1986). Elder Abuse \& Neglect: Causes, Diagnosis \& Intervention Strategies. New York: Springer Publishing Co.

REIS, M., D. NAHMIASH et R. SHRIER (1993, octobre). A brief abuse screen for the elderly (BASE): : Its validity and use. Paper presented at the 22nd Annual Scientific \& Educational Meeting of the Canadian Association on Gerontology, Montréal, Québec. 
Reis, M., D. Nahmiash, R. Shrier et C. SenNeville (1993, novembre). Indicators which discriminate abuse from nonabuse of the elderly. The 46th Annual Scientific Meeting of the Gerontological Society of America, New Orleans.

REIS, M. et D. NAHMIASH (à paraître en 1996). "When seniors are abused: An intervention model », The Gerontologist, vol. 35.

REIS, M. et D. NAHMIASH (1995). When seniors are abused: A guide to intervention. Toronto: Captus Press.

ROCHON, M. (1994). "Caractéristiques socio-démographiques des aînés du Québec et vieillissement de la population», dans P. Caris et B. Mishara (dir.), L'avenir des aînés au Québec après I'an 2000, p. 217-287. Québec: Presses de I'Université du Québec.

ROSENTHAL, C.J. (1987). "Aging and intergenerational relations in Canada", dans V.W. Marshall (dir.), Aging in Canada. Markham, Ont. : Fitzhenry \& Whiteside, $2^{\mathrm{e}}$ éd.

SANTÉ CANADA (1993). Older Canadians and the abuse of seniors: A continuum from participation to empowerment. Ottawa: Division de la prévention de la violence familiale.

SenGSTOCK, M.E. et M. HWALEK (1985). Comprehensive Index of Elder Abuse. Detroit, MI: Wayne State University.

SHELL, D.J. (1982). Protection of the Elderly: A Study of Elder Abuse. Winnipeg, Manitoba: Manitoba Council on Aging (Association on Gerontology).

Stevenson, C. (1985). Family abuse of the elderly in Canada. Report for the Senior Citizens Bureau. Alberta Social Services and Community Health.

StOne, R., G.L. Cafferata et J. SANGl (1987). "Caregivers of the frail elderly: A national profile», The Gerontologist, vol. 27, n 5 : 616-626.

StONES, M. (1991). A lexicon for elder mistreatment. Paper prepared by the Provincial Working Committee on Elder Mistreatment, St. John, Newfoundland.

STRYCKMAN, J. et D. NAHMIASH (1994). "Payment for care: The case of Canada», dans A. Evers, M. Pijl et C. Ungerson (dir.), Payment for Care: A comparative overview. European Center, Vienna: Avebury.

SYNGE, J. (1980). "Work and family support patterns of the aged in the early twentieth century, dans V.W. Marshall (dir.), Aging in Canada: Social Perspectives, p. 135-144. Don Mills, Ont.: Fitzhenry \& Whiteside.

THÉRIAULT, C. (1994). Inventaire des perceptions des personnes âgées de 70 ans et plus fréquentant un centre de jour, sur la violence exercée envers les personnes âgées (Territoire du CLSC du Centre-de-la Mauricie). Mémoire présenté pour l'obtention du grade de maître en service social. Québec: Université Laval, École de service social.

U.S. CONGRESS (1991). Physical and Financial Abuse of the Elderly. Washington, DC: Health Select Committee on Aging, United States Government Printing Office.

WOlF, R. et K.A. PILlemer (1994). "What's new in elder abuse programming? Four bright ideas », The Gerontologist, vol. 34, $\mathrm{n}^{\circ} 1$ : 126-129. 